\title{
Local Robust Gradient Patterns for Recognition of Cardiomyopathy
}

\author{
P.Megana Santhoshi, Mythili Thirugnanam
}

\begin{abstract}
Cardiomyopathy is one of the heart diseases that cause chamber damages. The impact of heart disease ends up in unforeseen fall with light-headedness. IoT plays an important role in human healthcare systems. Through IoT, it's terribly simple to watch the health condition of the heart disease patient by detection the abnormality within the electrocardiogram signal generated by IoT sensors. The varied ECG signals represent the severity of the heart disease and every graphical record signal has distinctive patterns. This paper describes the recognition of cardiomyopathy disease based on local robust gradient patterns technique LBP operator is one of the foremost powerful techniques to recognize the patterns within the ECG graph signals. But it's highly sensitive to noise and little fluctuations. To beat these limitations LTP and its derivatives are applied. LTP operator removes the noise by dividing the signals into 3 regions. It doesn't provide fruitful results if the signal has an additional range of peaks and valleys. Merely it replaces peaks by the valley and vice-versa. RLTP technique is appropriate to beat this limitation by finding the minimum value of LTP and its complement value. However, it fails for little fluctuation in the signals. To enhance the recognition rate of little fluctuation graphical record signals the discriminant robust local ternary pattern technique is proposed by multiplying the edge gradient values with RLTP techniques. This method is applied to PTB information and therefore the Experimental results are created within the variety of tables and graphs. The proposed technique has high results on the LTP and its derivative methods and is useful for detecting cardiomyopathy with $85 \%$ accuracy.
\end{abstract}

Keywords: LBP, LTP, RLTP, DRLTP, Cardiomyopathy, PTB, DCM, HCM.

\section{INTRODUCTION}

Cardiomyopathy is one of the heart diseases that cause chamber damages. In this disease heart muscles becomes dilated and thickened which leads to difficulties in pumping the blood. DCM (dilated cardiomyopathy), HCM (hypertrophic cardiomyopathy), RCM (restrictive cardiomyopathy), and ARVC (arrhythmogenic right ventricular cardiomyopathy) are four different types of

Revised Manuscript Received on December 30, 2019.

* Correspondence Author

P.Megana Santhoshi,* PhD Scholar, School of Computer Science Engineering, (SCOPE), Vellore Institute of Technology, Vellore, Tamil nadu, India. Email: Pmsanthoshi@Gmail.Com

Mythili Thirugnanam, Associate Professor Senior, School of Computer Science Engineering, (SCOPE), Vellore Institute of Technology, Vellore, Tamilnadu, India.

(C) The Authors. Published by Blue Eyes Intelligence Engineering and Sciences Publication (BEIESP). This is an open access article under the CC BY-NC-ND license (http://creativecommons.org/licenses/by-nc-nd/4.0/) cardiomyopathy, which are classified according to the affected Ventricular shape (dilated and thickened) and position (left and right) [5]. Dilated cardiomyopathy occurs when the left ventricle is enlarged, weak, and dilated. Hypertrophic cardiomyopathy occurs when the heart muscle (myocardium) becomes thickened; hence left ventricle valve becomes small. Restrictive cardiomyopathy occurs when the walls of the lower chambers of the heart (ventricles) are unusually rigid and lack the flexibility needed to expand as they fill with blood [13]. ARVC occurs if the muscle tissue in the right ventricle dies and is replaced with scar tissue [14]. The impact of heart disease ends up in unforeseen fall with light-headedness. IoT plays an important role in human healthcare systems [11]. Through IoT, it's terribly simple to watch the health condition of the heart disease patient by detecting the abnormality within the electrocardiogram (ECG) signal generated by IoT sensors.

An electrocardiogram signal contains the all information about heart conditions. Cardiomyopathy can also diagnose through ECG. The varied ECG signals represent the severity of the heart disease and every graphical record signal has distinctive patterns. An ECG signal represents the graphical illustration of the electrical activity of heart over an amount of time that is recorded by electrodes connected to the body with 12-leads or 3-leads. This ECG signal contains the P, QRS, $T$ and $U$ waves, which represent the atria and ventricular depolarization and repolarization [12]. Many of the researchers have been focused on P, QRS and T wave intervals, RR interval, PR \& QT intervals and ST, TP segments etc. to differentiate the disease.

In the differentiation (classification) of disease, one of the crucial steps is proper feature extraction. Good feature also simplifies the classification. This paper brings the concept of Local ternary patterns (LTP) and its derivatives (RLTP \& DRLTP) to extract the ECG features. The LTP patterns are the binary bits represented by either \pm 1 or 0 . These binary bits are converted into decimals which are referred as bins. These bins are used to represent the classification parameters to classify the disease. This paper refers only to the classification of two sections of cardiomyopathy. This categorizes DCM and HCM ECG signals with the normal ECG signal.

The rest of this paper is organized as follows. Section 2 briefly reviews the literature. Section 3 presents the proposed methods and section 4 classification parameters. In section 5 , the experimental results of three types of methods on three types of signals are reported and discussed. Finally, Section 6 concludes the paper.

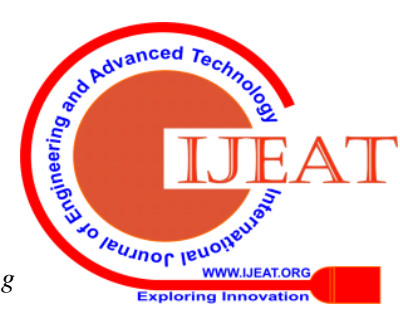




\section{LITERATURE SURVEY}

Based on analysis of ECG signal and classification techniques various automated systems have been proposed to classify cardiovascular diseases. Among these systems, ECG signals are analyzed by extracting features from wave $(\mathrm{P}$, QRS and T wave intervals,

RR interval, PR \& QT intervals and ST, TP segments etc.) and these features are time-based features, frequency-based features and wavelet-based features. Many classification techniques, such as Artificial Neural Network, Support Vector Machine, Neuro-Fuzzy, Hidden Markov Model, K-Nearest Neighbors, Genetic Algorithm etc., are proposed to classify the features and automatically differentiate the diseases. R. Acharya U et al [1] presented a system based on heart rate signal which classifies the cardiac diseases with the neural network and fuzzy classifiers. Here classified cardiac diseases are atrial fibrillation (AF), left bundle branch block (LBBB), ischemic/ dilated cardiomyopathy, normal sinus rhythm (NSR), sick sinus syndrome (SSS), pre-ventricular contraction (PVC), complete heart block (CHB) and ventricular fibrillation (VF). Due to limited training set and single input feature, this system achieved the low accuracy between $80-85 \%$. Digvijay Ghosh et al [2] Proposed an automatic system to detect and classify the cardiac abnormality through ECG signals. This system started with the pre-processing of ECG signals by filtering. Continuous wavelet Transform changed the time-based ECG signals to time-frequency signals and generated the CWT coefficients as feature vectors for appropriate disease. Based on this feature vector, SVM classified the various cardiac abnormalities like Dysrhythmia, Cardiomyopathy, Myocardial infarction, Myocardial Hypertrophy, and Valvular. This proposed system took the limited training sets for testing. Mirela Ovreiu and Dan Simon [3] Presented a system which detects the cardiomyopathy based on P-wave morphological features. This system achieved the $60 \%$ accuracy in classification of cardiomyopathy by applying Bio-geographical optimization to neuro-fuzzy training network. Shazwani Ahmad Shufni and M. Yusoff Mashor [4] was implemented statistical feature based system to classify the Myocardial infarction from normal and other diseased ECG waves. In this, multilayer perceptron classified the three types of domain features. Mean variance and standard deviation features were extracted in the time domain. The maximum point, minimum point, mean, variance, and standard deviation features were extracted in the frequency domain. In DWT, standard deviation feature only extracted from ECG waves. Only Limited data sets had been tested and shown that the more accuracy from DWT feature other than time and frequency domain was achieved.

Rabiya Begum et al [5] proposed an automated system to detect cardiomyopathy. In the first phase, the proposed system applied the pre-processing techniques to remove noise from ECG signal. In the second phase, system extracted the four time-based parameters such as PR, QRS, QT and RR intervals as features. In the last phase, extracted features were given to SVM and Feed forward back propagation (ANN) classifiers to categorize the disease from healthy subjects. The result shows that the ANN gave the better accuracy than SVM. Saksham Agarwal et al [6] illustrated the qualitative analysis for analyzing the ECG data to determine the seven types of cardiovascular diseases using the wavelet coherence and S-Transforms plots. In this analysis, time series based fluctuation measurements was used to differentiate the diseases, but with no performance results.

R. K. Tripathy and S. Dandapat [7] proposed a method to automatically detect cardiac abnormalities based on multiscale phase alternative features from multi-lead ECG waves. In this, ECG signals was translated into waveforms by using dual-tree complex wavelet transform (DTCWT) to extract the features from different scales. This method demonstrated that the fuzzy KNN has achieved more accuracy than the KNN in classifying PA features.

U. Rajendra Acharya et.al [8] implemented convolutional neural network algorithm to automatically detect the myocardial infarction based on ECG beats.11-layer CNN was used to detect the abnormal ECG beat without any feature selection. This approach had given the efficient performance at ECG beat without noise than at ECG beat with noise. But in this approach for training the network, requires huge data. Válber César Cavalcanti Roza et al [9] presented a design to detect the arrhythmia dataset (AD) and supraventricular arrhythmia dataset (SAD) from four time-based ECG features. An artificial neural network was used for disease classification with four input neurons, eight hidden neurons, and two output neurons. Due to classification mistakes in patterns of arrhythmias, this design achieved the less accuracy. Ashly Elizabeth Vincent and Sreekumar K [10] in their review paper investigated on ECG signal analysis with mainly aimed at feature extraction and classification approaches. And concluded that the fuzzy-c-means algorithm analyses the ECG signal is the efficient method. Based on the above study, most of the systems are classified myocardial infarction and types of arrhythmias accurately. Very few systems only concentrated on cardiomyopathy and achieved less accuracy. Automated classification of cardiomyopathy types (dilated and hypertrophic cardiomyopathy) has not been effectively implemented. Additionally, we found that one popular feature extraction technique: local pattern description has not been applied to extract ECG features yet. Since, this method can also extract ECG features with high accuracy. We adapted local ternary patterns (LTP) and its derivatives (RLTP and DRLTP) to extract the ECG features to classify the cardiomyopathy. These methods will be described in next section.

\section{PROPOSED METHOD}

To improve the classification rate of cardiomyopathy disease using ECG signal adapted the local pattern description methods. These methods are useful to extract the ECG features. In local pattern methods, LBP (local binary pattern) operator is one among the foremost powerful techniques to recognize the patterns within the ECG graph signals. This LBP operator divides signal into frames. For every frame, it encodes the data of the electrocardiogram signal as binary numbers by comparison anchor value (center value) with its four previous and succeeding values, and assigns them to zero or one. If those eight values exceed the threshold (center value), their binary codes are 1 ; if they're not or adequate the threshold, their binary codes are zero. It is formulated as follows, 


$$
L B P(Q)=\sum_{p=0}^{7} 2^{p} y\left(Q_{a}-Q_{n}\right), \quad y(x)=\left\{\begin{array}{ll}
1, & \text { if } x \geq 0 \\
0, & \text { if } x<0
\end{array}\right\}
$$

Where $\mathrm{Q}_{\mathrm{a}}$ is the Anchor value or center value and $\mathrm{Q}_{\mathrm{n}}$ is the neighbor values. This technique is easy however it having the constraints of high sensitivity to noise, micro patterns aren't recognized, intra class variation can't be reduced. These limitations are occurring because of LBP operator cannot differentiate the two values, one is nearer and slightly bit higher than the anchor value and another is nearer and tiny bit below the anchor value. And it's not appropriate for low contrasted electrocardiogram signal. To beat these limitations LTP (Local Ternary patterns) and its derivatives (RLTP \& DRLTP) are proposed.

LTP works as same as LBP in comparing the anchor value with previous and succeeding values. At last the difference comes in assigning the value codes. LBP assigns the two value code $(0,1)$ and LTP assigns the three value code $(-1,0$, 1). It reduces the noise which occurs in LBP. This method compares the ECG signal value with user threshold value and it assigns the value code. It is formulated as follows,

$$
\operatorname{LTP}(Q)=\sum_{p=0}^{7} 2^{p} y\left(Q_{a}-Q_{n}\right), \quad y(x)=\left\{\begin{array}{cc}
1, & \text { if } x \geq t \\
0, \text { if }-t<x<t \\
-1, & \text { if } x<-t
\end{array}\right\}
$$

Where $t$ is the user threshold, $Q_{a}$ is the Anchor value or center value and $Q_{n}$ is the neighbor values. With this method only noise problem is resolved. However to remove the other limitations, one of the LTP derivative i.e. RLTP is proposed. In this paper, minimum of LTP and its inversion is taken as Robust LTP (RLTP). This method overcomes the intra class variation. It is formulated as follows,

$$
\operatorname{RLTP}(Q)=\operatorname{minimum}\{\operatorname{LTP}(Q), \operatorname{LTP}(Q)\}
$$

Where $\operatorname{LTP}(Q)$ the complement of LTP (Q) .RLTP technique is is appropriate to beat this limitation of LTP. However, it fails for little fluctuation in the signals.

To enhance the recognition rate of little fluctuation graphical record signals the Discriminative robust local ternary pattern (DRLTP) technique is proposed by multiplying the edge gradient values with RLTP techniques. It formulated as follows,

$$
\begin{aligned}
& \operatorname{DRLTP}(Q)=D(Q) \times \operatorname{RLTP}(Q) \\
& D(Q)=\sqrt{D_{x}^{2}+D_{y}^{2}}
\end{aligned}
$$

Where $D_{x}=$ horizontal edges and $D_{y}=$ vertical edges. Edge gradient values computed with second order canny edge filter by applying the appropriate threshold values to improve the edge strength. DRLTP method overcomes the all limitations of LBP, LTP and RLTP methods.

\section{CLASSIFICATION PARAMETERS}

\section{A. MSE (Mean Square Error) \& PSNR (Peak Signal to Noise Ratio):}

PSNR value will be calculated with the assistance of Mean sq. error (MSE). Each square accustomed measure the standard rate before noise removal and after noise removal of the signals. PSNR and MSE square measure inter-linked and drawn from the ideas of signal process. The MSE formula outline as follows

$$
\text { MSE }=\frac{1}{n * m} \sum_{i=0}^{n-1} \sum_{j=0}^{m-1}\left[I\left(i_{i} j\right)-Q\left(i_{i} j\right)\right]^{2}
$$

Where I and Q are the ECG signal input and ECG query signal within the sort of an array with size of $n * m$ severally. MSE value is higher, the recognition rate is low and contrariwise.

PSNR outlined as

$$
P S N R=10 \cdot \log _{10}\left(\frac{256^{2}}{M S E}\right)
$$

Where PSNR value is higher the recognition rate is high and contrariwise. The PSNR value depends on MSE value log operate.

\section{B. Standard Deviation:}

Standard deviation is employed to calculate the variation among the signal values. If lower the SD values then the matching rate is high. I $(i, j)$ and $Q(i, j)$ are the input ECG signal and query ECG signal of size $\mathrm{n} * \mathrm{~m}$, whose standard deviation will be calculated as follows,

$$
S D=\sqrt{\frac{1}{n * m} \sum_{i=1}^{n} \sum_{j=1}^{m}((I(i, j)+Q(i, j))-\mu)^{2}}
$$

Where $n, m$ is the number of rows and columns of the signal matrix and $\mu$ is that the mean of the signal that is calculated as follows,

$$
\mu=\frac{1}{n * m} \sum_{i=1}^{n} \sum_{j=1}^{m}(I(i, j)+Q(i, j))
$$

\section{Jaccard index:}

The Jaccard index could be a statistical procedure wont to calculate the similarity rate between the input and output signals. This parameter is computed by applying division operation between intersection variables and union variables therefore it's also known as Intersection over Union. The Jaccard index worth lies between zero and one. If Jaccard worth is zero then the two signals are isolated and worth is one robust similarity between two signals.

$$
\mathrm{J}=\mathrm{S} 1 /(\mathrm{S} 1+\mathrm{S} 2)
$$

Wherever S1= (input electrocardiogram signal $\cap$ Query electrocardiogram signal),

S2 = (input electrocardiogram signal $\cup$ Query electrocardiogram signal).

In this paper, we've computed the Jaccard index to seek out the similarity between electrocardiogram signals.

\section{EXPERIMENTAL RESULTS}

The experimental results of LTP, RLTP, and DRLTP are generated on PTB info consists of two hundred cardiogram signals. For our experiments, we consider 21 ECG signals on three types of databases (DCM, HCM \& NORMAL). The DCM signal having a mild severity of disease, HCM signal is having high austerity of the disease. We have taken the ECG signal through IoT sensors and compared with these three types of query ECG signals.

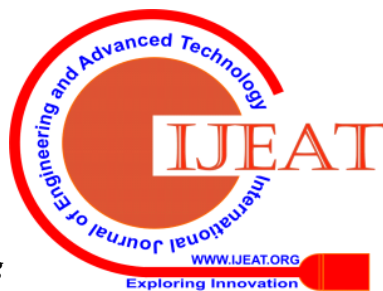


The parameters like MSE, standard deviation, PSNR and Jaccard formula are used to detect the ECG signal which is taken from the IoT sensor. Here we took three ECG signals from IoT sensors and consider this as query signals. Among these three ECG signals, Table 1to table- 4 and graph 1 to graph 4 shows that the signal is DCM, Table 5to table 8 and graph 5 to graph 8 shows that the second signal is HCM, and Table 9to table 12 and graph 9 to graph 12 shows that the signal is NORMAL. Table 13 shows that accuracy rate of classification on LTP, RLTP, and DRLTP.

The graph and table show the detection rate of the signal, and the query signal was classified as a DCM signal and a second query ECG signal is taken from the IoT sensor and it is found to be the HCM signal, which is high austerity. Third ECG signal is taken from the IoT sensor and recognize it as a normal ECG signal.

Table 13: Accuracy rate of classification on LTP, RLTP and DRLTP.

\begin{tabular}{|l|l|l|ll|}
\hline & MSE & $\begin{array}{l}\text { Standard } \\
\text { deviation }\end{array}$ & PSNR & Jaccord \\
\hline LTP & $71.4 \%$ & $42.8 \%$ & $71.4 \%$ & $71.4 \%$ \\
\hline
\end{tabular}

\begin{tabular}{|lllll|}
\hline RLTP & 14.2 & $42.8 \%$ & $28.5 \%$ & $71.4 \%$ \\
\hline DRLTP & $85.4 \%$ & $57.1 \%$ & $85.2 \%$ & $85.7 \%$ \\
\hline
\end{tabular}

Comparatively the accuracy rate of the proposed method is 85\%. It is higher than LTP and RLTP.

\section{CONCLUSION}

In this work, LTP and its derivatives have been implemented and tested with Dilated and Hypertrophic cardiomyopathy ECG signals and normal ECG signal provided by the PTB database through online. According to the experimental result, between the LTP, RLTP, and DRLTP methods, DRLTP have shown the best performance by providing an accuracy rate of $85 \%$ and by eliminating all limitations of the LTP and RLTP methods. However this proposed DRLTP method classifies accurately when the signal has the severity of the disease, but for signals of low severity, its performance is poor. This method classifies the Mild DCM signal as a normal signal and the Mild HCM signal as a DCM signal. Further to improve the accuracy of the system this limitation will be taken as a future work.
Table 1: DCM signal MSE values

\begin{tabular}{|r|r|r|}
\multicolumn{1}{|l|}{ LTP } & \multicolumn{1}{l|}{ RLTP } & \multicolumn{1}{l|}{ RLLTP } \\
\hline 9 & 9.999 & 9 \\
\hline 6.5445 & 8.9194 & 6.40561 \\
\hline 8.6918 & 8.5226 & 5.63496 \\
\hline 7.93574 & 9.7596 & 7.3489 \\
\hline 8.6918 & 8.5226 & 5.63496 \\
\hline 8.6916 & 8.5992 & 5.76819 \\
\hline 8.2688 & 9.9599 & 4.4981 \\
\hline 8.657 & 9.9738 & 7.93441 \\
\hline 8.1418 & 8.8591 & 6.44652 \\
\hline 8.6918 & 8.5629 & 5.68311 \\
\hline 8.0455 & 8.935 & 6.82537 \\
\hline 7.8517 & 9.2299 & 7.66009 \\
\hline 8.6918 & 8.5226 & 5.63496 \\
\hline 8.6916 & 8.6163 & 5.86256 \\
\hline 8.46397 & 9.9819 & 4.5169 \\
\hline 6.3681 & 9.5591 & 3.6529 \\
\hline 8.908 & 9.998 & 6.62022 \\
\hline 8.6918 & 8.545 & 5.66044 \\
\hline 8.7867 & 8.5926 & 5.71957 \\
\hline 8.75614 & 9.9742 & 6.8198 \\
\hline 8.6918 & 8.5226 & 5.63496 \\
\hline & & \\
\hline
\end{tabular}

Table 2: DCM signal Standard deviation values

\begin{tabular}{|r|l|r|}
\hline \multicolumn{1}{|l|}{ LTP } & RLTP & \multicolumn{1}{l|}{ DRLTP } \\
\hline 285.2329 & 220.2803 & 51.9408 \\
\hline 191.0647 & 154.7278 & 31.8643 \\
\hline 142.6165 & 110.1402 & 25.9704 \\
\hline 263.2646 & 207.5918 & 55.2923 \\
\hline 142.6165 & 110.1402 & 25.9704 \\
\hline 167.5397 & 134.3844 & 27.7755 \\
\hline 293.1686 & 223.2473 & 55.6228 \\
\hline 291.078 & 223.1943 & 71.826 \\
\hline 202.462 & 161.0339 & 34.0241 \\
\hline 160.7273 & 127.9333 & 27.1859 \\
\hline 208.9749 & 167.2773 & 38.4254 \\
\hline 221.6532 & 177.5036 & 41.1507 \\
\hline 142.6165 & 110.1402 & 25.9704 \\
\hline 170.2657 & 137.0542 & 28.6091 \\
\hline 273.7285 & 218.6355 & 63.5367 \\
\hline 245.5737 & 194.0771 & 54.9012 \\
\hline 283.2854 & 220.9962 & 81.9882 \\
\hline 156.1081 & 123.6849 & 26.7467 \\
\hline 167.7383 & 134.4455 & 27.9115 \\
\hline 284.184 & 219.9544 & 67.4616 \\
\hline 142.6165 & 110.1402 & 25.9704 \\
\hline
\end{tabular}

Table 3: DCM signal PSNR values

\begin{tabular}{|r|r|r|}
\hline \multicolumn{1}{|l|}{ LTP } & \multicolumn{1}{|l|}{ RLTP } & \multicolumn{1}{l|}{ DRLTP } \\
\hline 45.3851 & 29.6633 & 44.4438 \\
\hline 41.1669 & 28.3051 & 43.6001 \\
\hline 53.1476 & 36.1907 & 55.7657 \\
\hline 41.1669 & 28.3051 & 43.6001 \\
\hline 41.1673 & 28.5363 & 43.7347 \\
\hline 42.3835 & 43.968 & 52.5949 \\
\hline 41.2809 & 45.8169 & 46.8496 \\
\hline 42.691 & 29.4277 & 44.4935 \\
\hline 41.1669 & 28.4251 & 43.6483 \\
\hline 42.9104 & 29.7265 & 44.9831 \\
\hline 43.321 & 31.1344 & 46.308 \\
\hline 41.1669 & 28.3051 & 43.6001 \\
\hline 41.1673 & 28.5896 & 43.8327 \\
\hline 51.864 & 47.4326 & 52.6097 \\
\hline 45.6013 & 33.5566 & 51.9743 \\
\hline 50.3822 & 56.9593 & 44.7111 \\
\hline 41.1669 & 28.3715 & 43.6255 \\
\hline 40.8395 & 28.5159 & 43.6851 \\
\hline 50.9477 & 45.8861 & 54.9754 \\
\hline 41.1669 & 28.3051 & 43.6001 \\
\hline
\end{tabular}


Table 4: DCM signal Jaccord formula values

\begin{tabular}{|r|r|r|}
\hline \multicolumn{1}{|l|}{ LTP } & \multicolumn{1}{l|}{ RLTP } & \multicolumn{1}{l|}{ DRLTP } \\
\hline 0.533 & 0.556 & 0.6013 \\
\hline 0.5691 & 0.5911 & 0.6305 \\
\hline 0.533 & 0.556 & 0.6013 \\
\hline 0.823 & 0.8322 & 0.8593 \\
\hline 0.89 & 0.79 & 0.59 \\
\hline 0.5443 & 0.5677 & 0.6111 \\
\hline 0.7735 & 0.7961 & 0.8358 \\
\hline 0.7691 & 0.7918 & 0.8206 \\
\hline 0.5774 & 0.5995 & 0.6382 \\
\hline 0.5395 & 0.5615 & 0.6058 \\
\hline 0.5809 & 0.6032 & 0.641 \\
\hline 0.737 & 0.7198 & 0.7788 \\
\hline 0.533 & 0.556 & 0.6013 \\
\hline 0.5496 & 0.5722 & 0.6155 \\
\hline 0.7871 & 0.8014 & 0.833 \\
\hline 0.8714 & 0.882 & 0.8846 \\
\hline 0.7809 & 0.8019 & 0.8277 \\
\hline 0.5372 & 0.5605 & 0.6049 \\
\hline 0.5434 & 0.5668 & 0.61 \\
\hline 0.7778 & 0.7906 & 0.8265 \\
\hline 0.533 & 0.556 & 0.6013 \\
\hline
\end{tabular}

Table 7: HCM signal PSNR values

\begin{tabular}{|l|l|r|}
\hline LTP & RLTP & \multicolumn{1}{l|}{ DRLTP } \\
\hline 42.9104 & 29.7265 & 44.9831 \\
\hline 48.7343 & 59.7601 & 59.4529 \\
\hline 45.2474 & 44.5977 & 57.0514 \\
\hline 51.1183 & 35.3017 & 47.1789 \\
\hline 45.2474 & 44.5977 & 57.0514 \\
\hline 45.2478 & 46.2002 & 57.4966 \\
\hline 46.3915 & 28.2043 & 42.2346 \\
\hline 45.3681 & 28.4842 & 39.9754 \\
\hline 40.0111 & 56.3719 & 59.5467 \\
\hline 45.2474 & 45.3989 & 57.2118 \\
\hline 40 & 60 & 60 \\
\hline 47.1573 & 46.0429 & 57.8 \\
\hline 45.2474 & 44.5977 & 57.0514 \\
\hline 45.2478 & 46.6064 & 57.8127 \\
\hline 53.7511 & 30.9013 & 42.2395 \\
\hline 41.5119 & 38.6451 & 49.4122 \\
\hline 52.5597 & 29.4627 & 38.926 \\
\hline 45.2474 & 45.0336 & 57.1362 \\
\hline 44.8716 & 46.0487 & 57.3338 \\
\hline 48.3152 & 31.1634 & 42.9842 \\
\hline 45.2474 & 44.5977 & 57.0514 \\
\hline
\end{tabular}

Table 10: Healthy signal Standard deviation values

\begin{tabular}{|r|r|r|}
\hline \multicolumn{1}{|l|}{ LTP } & RLTP & \multicolumn{1}{l|}{ DRLTP } \\
\hline 142.6165 & 110.1402 & 25.9704 \\
\hline 48.4482 & 44.5877 & 5.8939 \\
\hline 0 & 0 & 0 \\
\hline 120.6481 & 97.4516 & 29.3219 \\
\hline 0 & 0 & 0 \\
\hline 24.9232 & 24.2443 & 1.8051 \\
\hline 150.5522 & 113.1071 & 29.6524 \\
\hline 148.4616 & 113.0541 & 45.8556 \\
\hline 59.8455 & 50.8938 & 8.0537 \\
\hline 18.1109 & 17.7932 & 1.2155 \\
\hline 66.3585 & 57.1371 & 12.455 \\
\hline 79.0367 & 67.3634 & 15.1803 \\
\hline 0 & 0 & 0 \\
\hline 27.6493 & 26.9141 & 2.6387 \\
\hline 131.112 & 108.4953 & 37.5663 \\
\hline 102.9573 & 83.9369 & 28.9308 \\
\hline 140.669 & 110.856 & 56.0178 \\
\hline 13.4916 & 13.5448 & 0.7763 \\
\hline 25.1219 & 24.3053 & 1.9411 \\
\hline 141.5676 & 109.8143 & 41.4912 \\
\hline 0 & 0 & 0 \\
\hline & & \\
\hline
\end{tabular}

Table 5: HCM signal MSE values

\begin{tabular}{|r|r|r|}
\hline LTP & \multicolumn{1}{|l|}{ RLTP } & \multicolumn{1}{|l|}{ DRLTP } \\
\hline 8.0455 & 8.935 & 6.82537 \\
\hline 2.5281 & 9.9989 & 8.8657 \\
\hline 6.6524 & 9.9653 & 8.0282 \\
\hline 8.7063 & 9.705 & 8.08526 \\
\hline 6.6524 & 9.9653 & 8.0282 \\
\hline 6.6521 & 9.976 & 8.2203 \\
\hline 5.6434 & 8.4879 & 4.02219 \\
\hline 6.558 & 8.5823 & 8.99431 \\
\hline 8.9974 & 9.9977 & 8.89 \\
\hline 6.6524 & 9.9712 & 8.0997 \\
\hline 9 & 9.999 & 9 \\
\hline 4.8032 & 9.9751 & 8.3404 \\
\hline 6.6524 & 9.9653 & 8.0282 \\
\hline 6.6521 & 9.9782 & 8.3453 \\
\hline 7.62805 & 9.1874 & 4.02896 \\
\hline 8.5836 & 9.8634 & 8.85506 \\
\hline 8.19712 & 8.8683 & 8.719451 \\
\hline 6.6524 & 9.9686 & 8.0663 \\
\hline 6.9299 & 9.9752 & 8.1523 \\
\hline 3.2155 & 9.235 & 4.96986 \\
\hline 6.6524 & 9.9653 & 8.0282 \\
\hline
\end{tabular}

Table 8: HCM signal Jaccord formula values

\begin{tabular}{|r|r|r|}
\hline LTP & RLTP & \multicolumn{1}{|l|}{ DRLTP } \\
\hline 0.5809 & 0.6032 & 0.641 \\
\hline 0.1828 & 0.1883 & 0.1784 \\
\hline 0.1101 & 0.1124 & 0.1058 \\
\hline 0.6564 & 0.6621 & 0.6804 \\
\hline 0.9999 & 0.9999 & 0.9999 \\
\hline 0.1352 & 0.1388 & 0.1307 \\
\hline 0.5763 & 0.6095 & 0.6513 \\
\hline 0.5656 & 0.5924 & 0.608 \\
\hline 0.1894 & 0.1929 & 0.1821 \\
\hline 0.1223 & 0.1255 & 0.1169 \\
\hline 0.1101 & 0.1124 & 0.1058 \\
\hline 0.8432 & 0.8419 & 0.8411 \\
\hline 0.1101 & 0.1124 & 0.1058 \\
\hline 0.1391 & 0.1423 & 0.1349 \\
\hline 0.599 & 0.6099 & 0.6248 \\
\hline 0.7618 & 0.7639 & 0.7648 \\
\hline 0.5904 & 0.6096 & 0.6196 \\
\hline 0.1182 & 0.1217 & 0.1139 \\
\hline 0.1305 & 0.134 & 0.126 \\
\hline 0.5775 & 0.5846 & 0.6144 \\
\hline 0.1191 & 0.1124 & 0.1058 \\
\hline
\end{tabular}

\begin{tabular}{|c|c|c|}
\hline ITP & RLTP & DRLTP \\
\hline 8.6918 & 8.5226 & 5.63496 \\
\hline 7.9763 & 9.968 & 8.6163 \\
\hline 9 & 9.999 & 9 \\
\hline 7.41278 & 9.4722 & 7.14819 \\
\hline 9 & 9.999 & 9 \\
\hline 9 & 9.998 & 8.9894 \\
\hline 8.9101 & 8.003 & 2.41345 \\
\hline 8.9991 & 8.1117 & 8.787857 \\
\hline 6.8049 & 9.9773 & 8.5722 \\
\hline 9 & 9.9987 & 8.9986 \\
\hline 6.6524 & 9.9653 & 8.0282 \\
\hline 8.7333 & 9.8847 & 5.7673 \\
\hline 9 & 9.999 & 9 \\
\hline 9 & 9.9975 & 8.9688 \\
\hline 8.85393 & 8.823 & 2.42109 \\
\hline 4.2585 & 9.6945 & 8.12055 \\
\hline 2. 2691 & 8.4442 & 8.487497 \\
\hline 9 & 9.9989 & 8.9996 \\
\hline 9913 & 9.9981 & 8.9958 \\
\hline 8.34977 & 8.8804 & 3.48833 \\
\hline & 9.999 & 9 \\
\hline
\end{tabular}

Table 11: Healthy signal PSNR values

\begin{tabular}{|r|l|r|}
\hline LTP & RLTP & \multicolumn{1}{l|}{ DRLTP } \\
\hline 41.1669 & 28.3051 & 43.6001 \\
\hline 43.0615 & 44.9509 & 58.5897 \\
\hline 40 & 60 & 60 \\
\hline 54.1283 & 32.7757 & 45.4488 \\
\hline 40 & 60 & 60 \\
\hline 40 & 56.9463 & 59.9543 \\
\hline 40.374 & 26.9962 & 41.1996 \\
\hline 40.004 & 27.2392 & 39.1645 \\
\hline 45.0449 & 46.4336 & 58.4532 \\
\hline 40 & 58.9342 & 59.9941 \\
\hline 45.2474 & 44.5977 & 57.0514 \\
\hline 41.0266 & 39.3823 & 53.7339 \\
\hline 40 & 60 & 60 \\
\hline 40 & 55.9609 & 59.8666 \\
\hline 50.5921 & 29.2922 & 41.2039 \\
\hline 47.5903 & 35.15 & 47.2597 \\
\hline 48.8823 & 28.0806 & 38.203 \\
\hline 40 & 59.6428 & 59.9983 \\
\hline 40.0377 & 57.3281 & 59.9816 \\
\hline 52.1755 & 29.5095 & 41.8631 \\
\hline 40 & 60 & 60 \\
\hline
\end{tabular}

\begin{tabular}{|r|r|r|}
\hline \multicolumn{1}{|l|}{ LTP } & \multicolumn{1}{|l|}{ RLTP } & \multicolumn{1}{l|}{ DRLTP } \\
\hline 0.533 & 0.556 & 0.6013 \\
\hline 0.0818 & 0.0861 & 0.0818 \\
\hline 0.0011 & 0.0011 & 0.0011 \\
\hline 0.616 & 0.62 & 0.644 \\
\hline 0.9999 & 0.9999 & 0.9999 \\
\hline 0.0274 & 0.0287 & 0.0271 \\
\hline 0.5208 & 0.5578 & 0.6092 \\
\hline 0.511 & 0.5385 & 0.5605 \\
\hline 0.0917 & 0.0938 & 0.0886 \\
\hline 0.0136 & 0.0147 & 0.0125 \\
\hline 0.1101 & 0.1124 & 0.1058 \\
\hline 0.8214 & 0.8205 & 0.8209 \\
\hline $1 E-04$ & $1 E-04$ & $1 E-04$ \\
\hline 0.0326 & 0.0339 & 0.0325 \\
\hline 0.5483 & 0.5564 & 0.5771 \\
\hline 0.7248 & 0.726 & 0.7297 \\
\hline 0.5398 & 0.5601 & 0.5745 \\
\hline 0.0085 & 0.0097 & 0.0085 \\
\hline 0.0232 & 0.0246 & 0.0229 \\
\hline 0.5213 & 0.5293 & 0.5662 \\
\hline 0.0011 & 0.0011 & 0.0011 \\
\hline
\end{tabular}

Table 6: HCM signal Standard

\begin{tabular}{|r|r|r|}
\multicolumn{4}{|c|}{ deViation ValueS } \\
\hline \multicolumn{1}{|c|}{ LTP } & \multicolumn{1}{|l|}{ RLTP } & \multicolumn{1}{|c|}{ DRLTP } \\
\hline 208.9749 & 167.2773 & 38.4254 \\
\hline 114.8067 & 101.7248 & 18.3488 \\
\hline 66.3585 & 57.1371 & 12.455 \\
\hline 187.0066 & 154.5887 & 41.7769 \\
\hline 66.3585 & 57.1371 & 12.455 \\
\hline 91.2817 & 81.3814 & 14.26 \\
\hline 216.9107 & 170.2442 & 42.1074 \\
\hline 214.8201 & 170.1912 & 58.3106 \\
\hline 126.204 & 108.0309 & 20.5087 \\
\hline 84.4694 & 74.9303 & 13.6704 \\
\hline 132.717 & 114.2742 & 24.9099 \\
\hline 145.3952 & 124.5005 & 27.6353 \\
\hline 66.3585 & 57.1371 & 12.455 \\
\hline 94.0078 & 84.0512 & 15.0936 \\
\hline 197.4705 & 165.6324 & 50.0213 \\
\hline 169.3158 & 141.074 & 41.3858 \\
\hline 207.0275 & 167.9931 & 68.4728 \\
\hline 79.8501 & 70.6819 & 13.2313 \\
\hline 91.4803 & 81.4424 & 14.3961 \\
\hline 207.9261 & 166.9513 & 53.9462 \\
\hline 66.3585 & 57.1371 & 12.455 \\
\hline
\end{tabular}

Table 9: Healthy signal MSE values
Table 12: Healthy signal Jaccord formula values \begin{tabular}{|l|l|l|}
\hline 0.0011 & 0.0011 & 0.0011 \\
\hline
\end{tabular} 

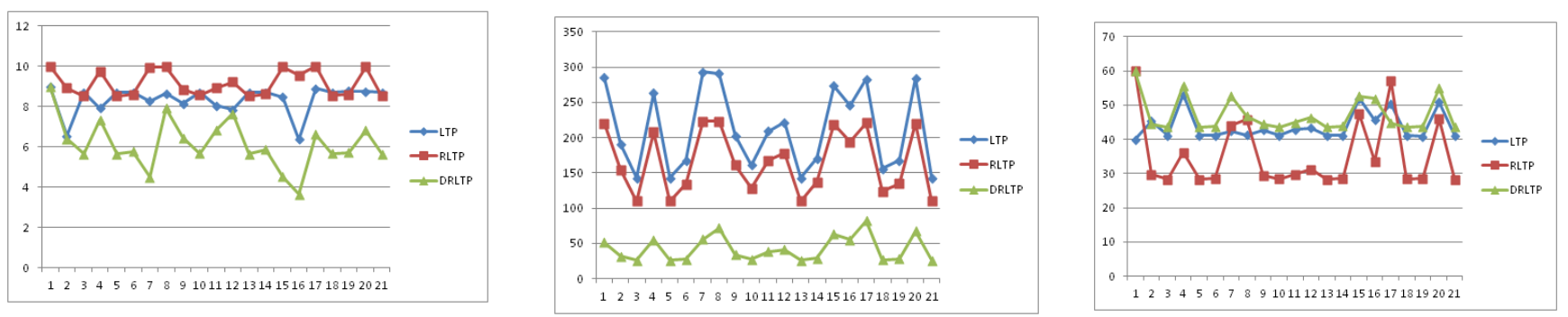

Graph 4: DCM signal Jaccord formula values

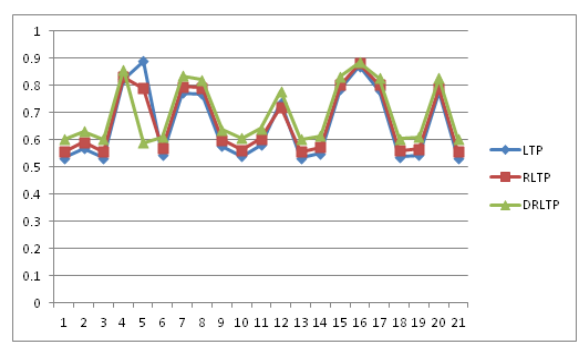

Graph 7: HCM signal PSNR values

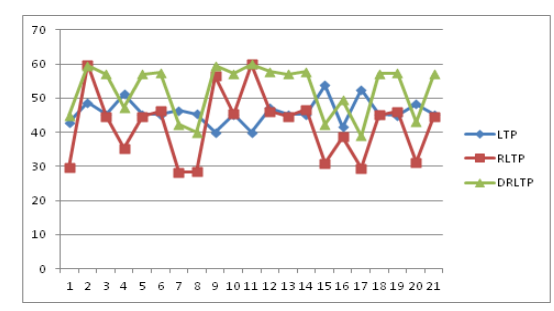

Graph 10: Healthy signal Standard deviation values

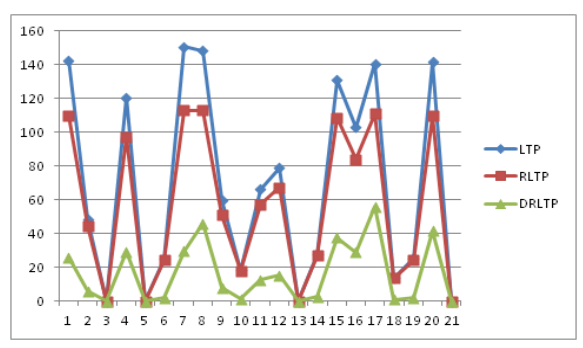

\section{REFERENCES}

1. R. Acharya, A. Kumar, P.S. Bhat, C.M. Lim, N. Kannathal, and S.M. Krishnan, "Classification of cardiac abnormalities using heart rate signals," Medical and Biological Engineering and Computing, vol.42, no.3, pp.288-293, 2004.

2. D. Ghosh, B. L. Midya, C. Koley, and P. Purkait, "Wavelet Aided SVM Analysis of ECG Signals for Cardiac Abnormality Detection," Annual IEEE India Conference - Indicon, pp. 9-13, 2005.

Published By:

Blue Eyes Intelligence Engineering

\& Sciences Publication
Graph 6: HCM signal Standard deviation values

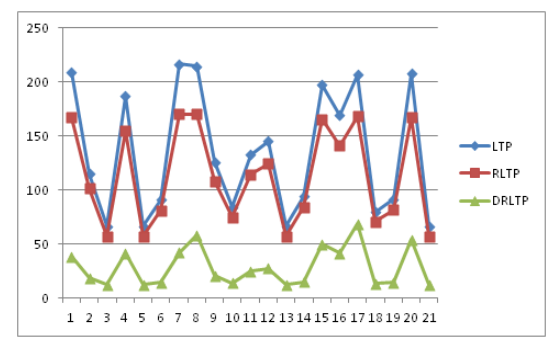

Graph 9: Healthy signal MSE values

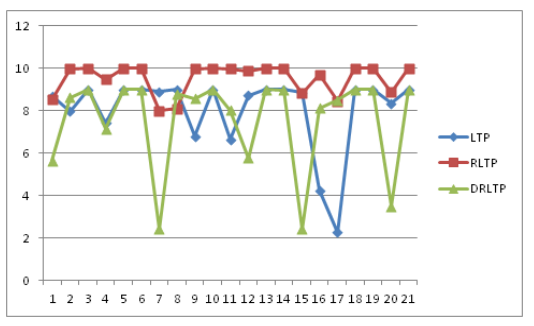

Graph 12: Healthy signal Jaccord formula values

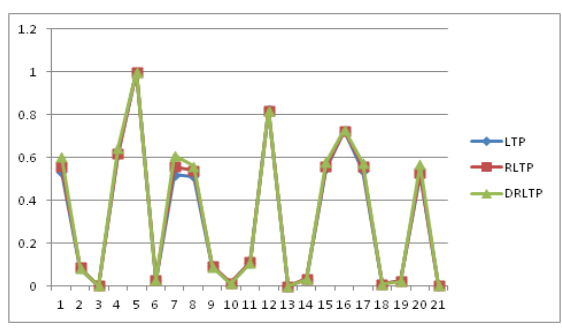

3. M. Ovreiu and D. Simon, "Cardiomyopathy Detection from Electrocardiogram Features," Cardiomyopathies - Basic Res. Clin. Manag, pp. 117-134, Feb. 2012.

4. S. A. Shufni and M. Y. Mashor, "ECG signals classification based on discrete wavelet transform, time domain and frequency domain features," 2nd International Conference on Biomedical Engineering (ICoBE), pp. 1-6, 2015.

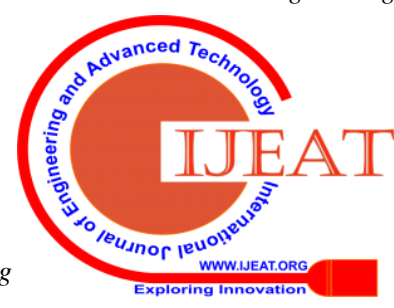


5. R. Begum and M. Ramesh, "Detection of Cardiomyopathy using Support Vector Machine and Artificial Neural Network," Int. J. Comput. Appl., vol. 133, no. 14, pp. 29-34, Jan. 2016.

6. S. Agarwal, V. Krishnamoorthy, and S. Pratiher, "ECG signal analysis using wavelet coherence and s-transform for classification of cardiovascular diseases," International Conference on Advances in Computing, Communications and Informatics (ICACCI), pp. 2765-2770, 2016.

7. R. K. Tripathy and S. Dandapat, "Detection of Cardiac Abnormalities from Multilead ECG using Multiscale Phase Alternation Features," $J$. Med. Syst., vol. 40, no. 6, p. 143, Jun. 2016.

8. U. R. Acharya, H. Fujita, S. L. Oh, Y. Hagiwara, J. H. Tan, and M. Adam, "Application of deep convolutional neural network for automated detection of myocardial infarction using ECG signals," Inf Sci., vol. 415-416, pp. 190-198, Nov. 2017.

9. V. C. C. Roza, A. M. de Almeida, and O. A. Postolache, "Design of an artificial neural network and feature extraction to identify arrhythmias from ECG," IEEE International Symposium on Medical Measurements and Applications (MeMeA), pp. 391-396, 2017.

10. A. E. Vincent and K. Sreekumar, "A survey on approaches for ECG signal analysis with focus to feature extraction and classification," International Conference on Inventive Communication and Computational Technologies (ICICCT), pp. 140-144, 2017.

11. J. Cubo, A. Nieto, \& E. Pimentel, "A cloud-based Internet of Things platform for ambient assisted living," Sensors, Vol.14, no.8, pp.14070-14105, 2014

12. B. Subramanian, "ECG signal classification and parameter estimation using multiwavelet transform," Biomedical Research, 2017.

13. L. Emily, A. Devon \& G. Mohan. ( 2019 ). Cardiomyopathy types: Dilated, hypertrophic, restrictive, ischemic, and alcoholic. [Online]. Available:

https://www.belmarrahealth.com/cardiomyopathy-types-dilated-hyper trophic-restrictive-ischemic-alcoholic/

14. Arrhythmogenic right ventricular cardiomyopathy, (2018, May, 10) [Online]. Available: http://www.cardiomyopathy.org/arrhythmogenic-right-ventricular-car diomyopathy/intro

\section{AUTHORS PROFILE}

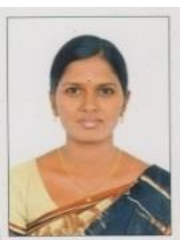

Megana Santhoshi Pasupuleti is a Research Scholar in the School of Computer Science and Engineering at Vellore Institute of Technology, Vellore, India. She received her Master's in Computer Science and Engineering from Annamacharya Institute of Technology \& Sciences, JNT University, Anantapur in 2011. Currently working as an Assistant Professor in the Department of Computer Science and Engineering at Chaitanya Bharathi Institute of Technology, JNT University, Anantapur. She has teaching experience of around Four years. Her area of specialization includes Biomedical Signal Processing, Machine Learning, and Internet of Things (IOT). She has published two papers in international journals.

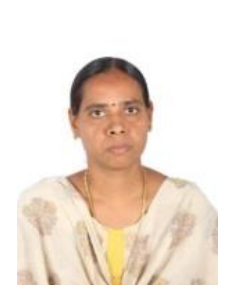

Mythili Thirugnanam is an Associate Professor Senior in the School of Computer Science and Engineering at Vellore Institute of Technology, Vellore, India. She received a Master's in Software Engineering from Vellore Institute of Technology. She has been awarded a doctorate in Computer Science and Engineering at Vellore Institute of Technology in 2014. She has teaching experience of around 12 years. She has research experience of 3 years in handling sponsored projects funded by Govt. of India. Her area of specialization includes Digital Image Processing, Software Engineering, Artificial Intelligence and Machine Learning. She has published more than 40 research papers in international and national journals and presented around 12 papers in various national and international conferences. 